\title{
Data resource profile: the National Health Insurance Research Database (NHIRD)
}

\author{
Liang-Yu Lin ${ }^{1,2}$, Charlotte Warren-Gash' ${ }^{1}$ Liam Smeeth ${ }^{1}$, Pau-Chung Chen ${ }^{2,3,4,5,6}$ \\ 'Department of Non-communicable Disease Epidemiology, Faculty of Epidemiology and Population Health, London School of Hygiene and \\ Tropical Medicine, London, UK; ${ }^{2}$ Institute of Occupational Medicine and Industrial Hygiene, National Taiwan University College of Public Health, \\ Taipei, Taiwan; ${ }^{3}$ Department of Public Health, National Taiwan University College of Public Health, Taipei, Taiwan; Innovation and Policy \\ Centre for Population Health and Sustainable Environment, National Taiwan University College of Public Health, Taipei, Taiwan; ${ }^{5}$ Department \\ of Environmental and Occupational Medicine, National Taiwan University College of Medicine and Hospital, Taipei, Taiwan; ${ }^{6}$ Office of \\ Occupational Safety and Health, National Taiwan University Hospital, Taipei, Taiwan
}

\begin{abstract}
Electronic health records (EHRs) can provide researchers with extraordinary opportunities for population-based research. The National Health Insurance system of Taiwan was established in 1995 and covers more than 99.6\% of the Taiwanese population; this system's claims data are released as the National Health Insurance Research Database (NHIRD). All data from primary outpatient departments and inpatient hospital care settings after 2000 are included in this database. After a change and update in 2016, the NHIRD is maintained and regulated by the Data Science Centre of the Ministry of Health and Welfare of Taiwan. Datasets for approved research are released in three forms: sampling datasets comprising 2 million subjects, disease-specific databases, and full population datasets. These datasets are de-identified and contain basic demographic information, disease diagnoses, prescriptions, operations, and investigations. Data can be linked to government surveys or other research datasets. While only a small number of validation studies with small sample sizes have been undertaken, they have generally reported positive predictive values of over $70 \%$ for various diagnoses. Currently, patients cannot opt out of inclusion in the database, although this requirement is under review. In conclusion, the NHIRD is a large, powerful data source for biomedical research.
\end{abstract}

KEY WORDS: Database, Electronic health records, Information storage and retrieval, National Health Insurance Research Database, Taiwan

\section{INTRODUCTION}

The increasing availability, size, and detail of electronic health records (EHRs) offer unprecedented opportunities for research. The advantages of EHRs include increased statistical power, speed, wide breadth, relatively low cost, representative population cover-

\section{Correspondence: Pau-Chung Chen}

Institute of Occupational Medicine and Industrial Hygiene, National Taiwan University College of Public Health, 17 Xu-Zhou Road,

Taipei 10055, Taiwan

E-mail: pchen@ntu.edu.tw

Received: Nov 15, 2018 / Accepted: Dec 27, 2018 / Published: Dec 27, 2018

This article is available from: http://e-epih.org/

(C) This is an open-access article distributed under the terms of the Creative Commons Attribution License (http://creativecommons.org/licenses/by/4.0/), which permits unrestricted use, distribution, and reproduction in any medium, provided the original work is properly cited.

(C) 2018, Korean Society of Epidemiology age, completeness of follow-up, and the ability to assess interventions in routine clinical care [1]. Linking EHRs to disease registries and other resources can further extend their utility. Meanwhile, randomised controlled trials (RCTs) control for known and unknown confounding factors; therefore, they are regarded as the gold standard for measuring the efficacy of interventions [2]. However, the true effectiveness of exposures may be influenced by many factors in a real-world setting, leading to a gap between efficacy and effectiveness. Consequently, real-world data, collected in non-RCT settings, are essential to bridging this gap [3]. As an important source of real-world data, EHRs have become a practical tool in medical research. By utilising EHRs, researchers can measure treatment effects, demonstrate trends in disease incidence and prevalence, and further explore possible disease aetiologies.

Among national EHR databases all over the world, the National Health Insurance Research Database (NHIRD) of Taiwan is unique. This large database, which contains data from 23 million residents of Taiwan, was previously described by Chen et al. [4]. 
However, the NHIRD was updated in 2016. This database changed its regulatory administration, was integrated with other datasets for further linkage, and released its full population dataset. The NHIRD now provides greater flexibility for scientific research. In this article, we introduce the latest version of the NHIRD, demonstrate its key features for research, and describe its strengths and weaknesses.

\section{BASIC DATA RESOURCES}

\section{National Health Insurance programme of Taiwan}

To increase the affordability and accessibility of health care, in 1995, the Taiwanese government initiated a single-payer health insurance system, known as National Health Insurance (NHI). NHI has a contract with most healthcare facilities in Taiwan, and it is mandatory for physicians to upload the claims data from each visit to the National Health Insurance Ministry. Notably, the primary care system in Taiwan is different from that of many other countries. Referrals from general practitioners are not required to receive specialist care; therefore, patients with non-emergency health concerns can either visit local private or public clinics or go directly to specialists at hospital outpatient departments [5]. In 2017, 93\% of healthcare facilities in Taiwan contracted with NHI, except some self-pay private clinics [6]. As a programme that provides universal care health coverage, NHI covers all necessary medical expenses including outpatient visits, the inpatient system, prescriptions, treatment with traditional Chinese medicine, dental services, operations, and investigations such as X-rays or magnetic resonance imaging. The coverage of NHI reached $92 \%$ as it was established; by the end of 2014, NHI covered $99.9 \%$ of the Taiwanese population $[7,8]$.

\section{History of insurance data usage and governance}

In 2000, the anonymous and encrypted sampling dataset from this national insurance system was first released for use in research, under the regulation and maintenance of the National Health Research Institutes of Taiwan. From 2000 to 2013, the National Health Research Institutes made available to researchers

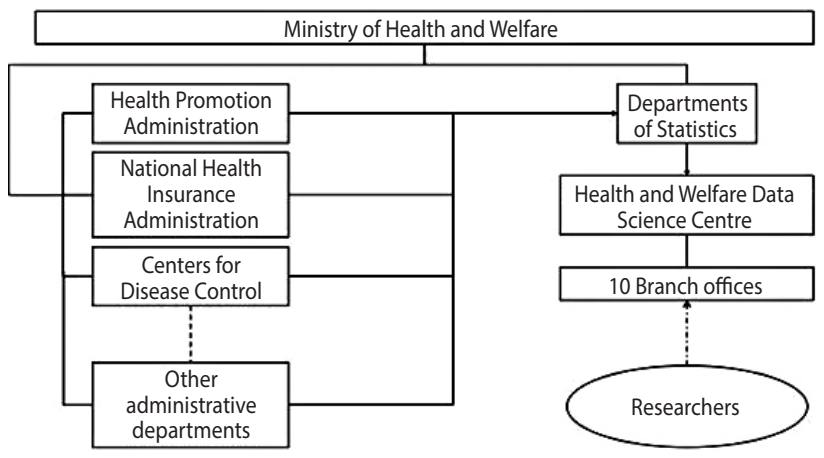

Figure 1. Administrative structure of the National Health Insurance Research Database [10]. general sampling datasets with 1 million subjects, as well as disease-specific sampling datasets. In 2016, these insurance data were moved to the Data Science Centre of the Ministry of Health and Welfare of Taiwan, where data are regulated and managed by the government [9]. The regulatory structure of the NHIRD is illustrated in Figure 1 [10]. The claims data from the NHI are stored and processed at the Data Science Centre, along with other governmental surveys and datasets. At the Data Science Centre, the NHIRD and other datasets are compared with the Household Registry Record from the Ministry of the Interior for quality control. Variables, such as sex and dates, are examined to ensure accuracy and consistency across different years. All data are deidentified and encrypted to protect participants' privacy [11].

\section{Research using the National Health Insurance Research Database data}

The NHIRD is a powerful for observing chronic diseases and assessing the effects of treatments. For instance, hepatitis $\mathrm{B}$ virus (HBV) and hepatitis $\mathrm{C}$ virus ( $\mathrm{HCV}$ ) infections are relatively prevalent in Taiwan. Previous studies using the NHIRD demonstrated that the use of statins, medicines for decreasing low-density lipoprotein in the blood, was associated with a decreased incidence of hepatocellular carcinoma (HCC) in HBV and HCV patients [8,9]. Another study showed that the use of nucleoside analogues as antiviral treatments for chronic hepatitis B reduced HCC recurrence in HBV patients receiving liver resection [12]. Furthermore, the availability of data linkage makes it possible to conduct population-based studies of rare diseases. By using NHIRD data, Kuo et al. [13] demonstrated an increased heritable risk of systemic lupus erythematosus (SLE) and other autoimmune diseases among families of SLE patients. Since 2014, more than 300 published studies have used NHIRD data each year (Figure 2). To date, over 2,700 peer-reviewed studies have been published using NHIRD data, covering such topics as general medicine, multidisciplinary

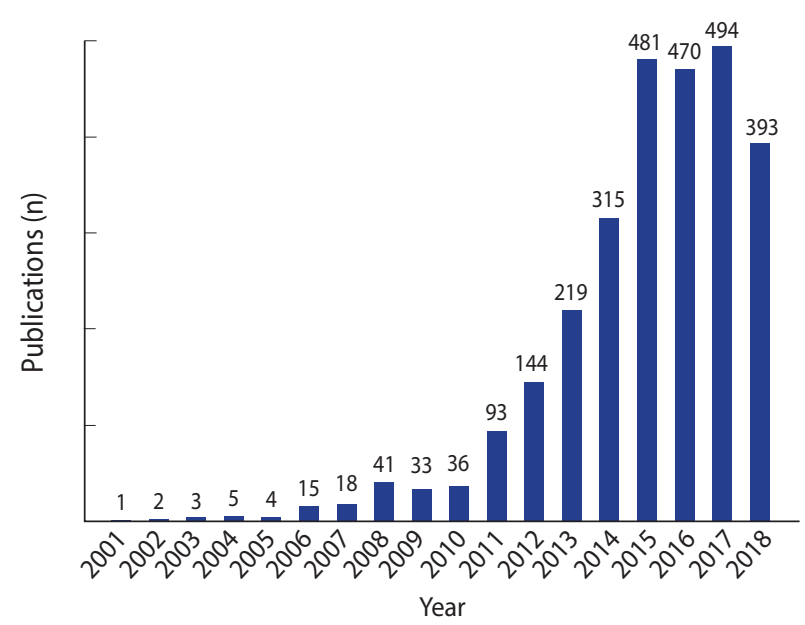

Figure 2. Publications using the National Health Insurance Research Database from 2000 to 2018. 
science, psychiatry, clinical neurology, oncology, and public environmental and occupational health.

\section{MEASUREMENTS}

\section{Practice and patient data}

The basic structure of the NHIRD data is shown in Figure 3. The de-identified data contain demographic variables, including the insured persons' registration location, sex, age, investigations, diagnoses, prescriptions, and details of each outpatient visit or their inpatient care. Disease diagnoses are coded using the International Classification of Diseases, Ninth Revision. Each subject in the dataset is coded with an encrypted identifier, which can be used to link future patient data. Detailed laboratory test results and medical notes are not included in this database.

\section{Data release}

The NHIRD data are released in three forms. The first form is a general dataset containing 2 million patients. Two million subjects are collected using stratified random sampling by age, sex, and the registry of regions from the full database population. They were sampled at three different time points: 2000, 2005, and 2010 (Supplementary Material 1). Each dataset contains claims data including diagnoses, prescriptions, investigation items, and treatments that the subjects received from 2000 to 2016 . For the datasets sampled in 2005 and 2010, two additional datasets are available: from 2005 to 2016 and from 2010 to 2016. In addition to the complete claims data, these sampling datasets also contain data from cause of death datasets, cancer registry datasets, major illness datasets, and hospital information datasets. The general 2-million-patient sampling dataset is considered to be nationally representative.

The second form of NHIRD data are disease-specific databases. These databases contain complete claims data of all patients with a certain health condition. For instance, all patients with a diabetes diagnosis from 2002 to 2015 are included in the diabetes data-

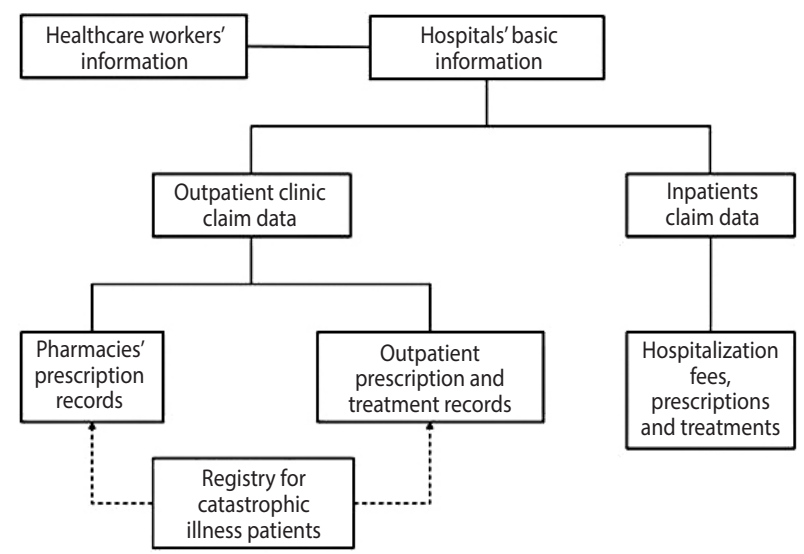

Figure 3. Data structure of the National Health Insurance Research Database. base. As of 2018, there are 13 disease-specific databases available for research (Table 1). These datasets can also be linked to cancer registry data and cause of death data.

The third form of NHIRD data is the full population dataset, which has been available for research since 2016 . The full population dataset covers the entire Taiwanese population from 2000 to 2016, which comprises approximately 23 million people. Researchers can apply for complete claims data, including inpatient and outpatient records, investigations, and treatment, which can be linked with hospital information, birth certificate applications, death records, the cancer registry dataset, and the major illness datasets. Furthermore, the full population data can also be linked with individual datasets, a feature that will be introduced later. These released datasets are a valuable source for epidemiological research.

\section{Data linkage}

Since 2016, under the authorisation and regulation of the Ministry of Health and Welfare, NHIRD data can be more widely linked with other public surveys at the Data Science Centre. These datasets include governmental surveys, disease registries, health surveys, social reporting system data, and welfare registry data. Detailed descriptions of these databases are given in Table 2. These databases and the NHIRD can be linked through an encrypted personal ID using deterministic record linkage. Due to privacy issues, this data linkage can only be processed by researchers at the Data Science Centre. Accessing some sensitive data, such as the domestic violence database, requires special authorisation from other administrative departments. In addition to linking governmental data, with the informed consent of study subjects, researchers are also allowed to link their own research databases with the NHIRD. For instance, the Taiwan Biobank Database is a national cohort containing biological samples and

Table 1. Disease-specific databases of the National Health Insurance Research Database

\begin{tabular}{lcr} 
Database name & Year & \multicolumn{1}{c}{$\begin{array}{c}\text { No. of new } \\
\text { case }\end{array}$} \\
\hline Colorectal Cancer Health & $2002-2015$ & 175,405 \\
Breast Cancer Health & $2002-2015$ & 136,476 \\
Prostate Cancer Health & $2002-2015$ & 53,937 \\
Systemic Lupus Erythematosus Health & $2002-2015$ & 29,637 \\
Hypertension Health & $2002-2015$ & $3,342,827$ \\
Brain Tumour Health & $2002-2015$ & 10,267 \\
Chronic Kidney Disease Health & $2002-2015$ & $1,066,892$ \\
End-Stage Renal Disease Health & $2002-2015$ & 134,228 \\
Diabetes Mellitus Health & $2002-2015$ & $1,720,602$ \\
Injury & $2000-2015$ & $25,925,939$ \\
Triple-High' & $2001-2015$ & 6,558 \\
Disability Process & $1996,1999,2003$, & 6,935 \\
& 2007,2011 & \\
Maternal and Child Health & $2004-2014$ & $2,171,765$ \\
\hline
\end{tabular}

${ }^{1}$ Triple-High: hypertension, hyperglycaemia, hyperlipidaemia. 
Table 2. Databases available for linkage

\begin{tabular}{|c|c|}
\hline Name of database & Year \\
\hline \multicolumn{2}{|l|}{ Health data } \\
\hline Taiwan Cancer Registry & $2007-2012$ \\
\hline Cause of death data & $1971-2014$ \\
\hline Birth certificate applications & $2001-2013$ \\
\hline Traffic accident data & $2003-2014$ \\
\hline "Triple-high Status" Survey & 2006-2007 \\
\hline Taiwan Birth Cohort Study & 2005 \\
\hline $\begin{array}{l}\text { "Knowledge, Attitude, and Practice of Contraception" } \\
\text { Survey }\end{array}$ & $1965-2008$ \\
\hline Taiwan Youth Health Survey File & $2006-2010$ \\
\hline Rare disease data & 2012 \\
\hline Artificial reproductive data & 1998-2012 \\
\hline Cancer screening - Pap smear data & 2004-2013 \\
\hline Colorectal cancer screening & $2010-2013$ \\
\hline Breast cancer screening & 2004-2013 \\
\hline Oral mucosal screening & $2010-2013$ \\
\hline $\begin{array}{l}\text { Taiwan Healthy Behaviour Risk Factor Surveillance } \\
\text { Survey File }\end{array}$ & $2007-2012$ \\
\hline \multicolumn{2}{|l|}{ Social surveys } \\
\hline National Aboriginal Population Profile & $2006-2012$ \\
\hline Personal data for the sampled NHI claims cohorts & 2000,2005 \\
\hline National Health Interview Survey & $2001-2009$ \\
\hline Taiwan Longitudinal Study on Aging & 1998-2011 \\
\hline Taiwan Smoking Behaviour Survey & 2004-2009 \\
\hline \multicolumn{2}{|l|}{ Welfare databases } \\
\hline The Juvenile Condition Survey in Taiwan-Fuchien Area & 2003 \\
\hline $\begin{array}{l}\text { Report of the Home Care Subsidy User Condition } \\
\text { Survey }\end{array}$ & 2007 \\
\hline The Satisfaction with Home Care Services Survey & 2011 \\
\hline $\begin{array}{l}\text { The Low-Income and Middle-Income Family Living } \\
\text { Condition Survey }\end{array}$ & 2013 \\
\hline Taiwan Longitudinal Study on Aging & $2009-2013$ \\
\hline $\begin{array}{l}\text { Physically and Mentally Disabled Citizens Living and } \\
\text { Demand Assessment Survey }\end{array}$ & 2011 \\
\hline Single Parent Family Condition Survey & 2010 \\
\hline Women's Living Conditions Survey & 1998-2011 \\
\hline Disabled Population Profile & 2014 \\
\hline Low-income and middle-low-income household data & 2014 \\
\hline Family violence data & 2011-2014 \\
\hline Reported data of protection of children and youths & 2011-2014 \\
\hline Reported data of sexual assault & 2011-2014 \\
\hline
\end{tabular}

$\mathrm{NHI}$, National Health Insurance.

comprehensive examinations of 200,000 adult volunteers that will be linked to the NHIRD [14]. Such data linkages can help researchers discover possible interactions among genes, environmental factors, and diseases.

\section{Strengths and weaknesses Strengths}

The NHIRD is a nationally representative cohort that contains detailed registry and claims data from all 23 million residents of Taiwan. This huge database provides researchers with powerful and generalisable real-world evidence for biomedical studies. For instance, a molecular epidemiological study has suggested that aristolochic acid (AA), an ingredient in Chinese herbal remedies, was correlated to HCC in Taiwan and other Asian countries [15]. Similar findings were later found using NHIRD data. Chen et al. [16] analysed NHIRD data and discovered that using Chinese herbs containing AA increased the risk of HCC among patients with HBV infections. In addition, after the update in 2016, the NHIRD can be further linked with other datasets to increase the power and potential to research specific population subgroups, rare conditions, and factors that are not usually contained in clinical databases, such as living conditions, violence, or detailed lifestyle data. Payment and reimbursement data are also valuable for health economic analyses.

\section{Weaknesses}

There are some issues with the NHIRD. First, the NHIRD lacks comprehensive validation, although some validation studies of the clinical diagnoses in the NHIRD have been done. Some of these validation studies used national disease registries as the reference standard, which is more convincing. Other studies used hospitalbased records to validate the diagnoses found in the NHIRD and have reported relatively high positive predictive values (over 70\%) (Supplementary Material 2). However, the samples of these studies were small and drawn from a limited number of hospitals. Therefore, the samples may not be regarded as nationally representative. To improve the accuracy of the NHIRD, the Ministry of Health and Welfare of Taiwan recently initiated a national validation project using existing registry data [17]. However, until this new evidence of the database's validity is reported, researchers should carefully interpret results from the NHIRD. Second, consent from the participants included in the NHIRD is another controversial issue. By law, all residents in Taiwan are required to have NHI, and their data are included in the NHIRD; there is currently no way for participants to opt out of this national cohort. However, in 2017, the Supreme Administrative Court upheld the legitimacy of using the NHIRD data for research [18]. People's ability to opt out of inclusion in the NHIRD remains under discussion [19]. Finally, records of self-pay healthcare and out-of-pocket payments, such as for cosmetic surgery, are not included in the NHIRD. This may narrow the scope of research using the NHIRD, and researchers must be aware of the effects of these non-included variables.

\section{Data access}

Researchers can access NHIRD data after ethical and scientific review processes. Prior to applying, researchers must obtain approval from the institutional review board. Notably, the applicant must be Taiwanese or be affiliated with a Taiwanese research institute. Applicants should submit their research proposal to the Data Science Centre. Proposals should include specific methods and variables required for their analyses. The cost of accessing data de- 
pends on the number of variables requested and the time period that they require the data for analyses; for example, accessing one variable for 1 year would cost 200 new Taiwanese dollars. After receiving an application, the Ministry of Health and Welfare reviews the legitimacy of the proposal, which is later reviewed by a scientific committee consisting of three experts. If one of the committee members disagrees with the proposed use of the data, then the researchers must submit a revised proposal to a higher advisory committee for a second review.

After receiving approval, researchers must go to the branches of the Data Science Centre to perform their data analyses. The analyses of NHIRD data are complicated, and there is no structural training course for using the NHIRD. Therefore, a mock dataset containing 100,000 subjects is provided by the Data Science Centre to help researchers in writing statistical analysis syntax. When researchers enter the Data Science Centre, they are allowed to use provided computers and software including SAS, Stata, R, and SPSS to conduct their data analyses [20].

\section{Ethics and confidentiality}

Ethical review board approval is mandatory when applying to use NHIRD data. There are 27 institutional review boards capable of issuing approvals, and all are supervised and regulated by the Ministry of Health and Welfare [21]. To protect individuals' confidentiality, all datasets in the Data Science Centre are pseudonymised. Personal ID, birth date, and names are encrypted, and this de-identification process was approved by an independent third party organisation [20]. To further secure the participants' privacy, NHIRD datasets cannot be accessed outside the Data Science Centre, meaning that researchers must analyse these datasets at the Data Science Centre. When accessing the Data Science Centre, researchers are not allowed to bring any recording devices, including paper and pen. In addition, their statistical analysis syntax needs to be reviewed by the Data Science Centre prior to using the computers and software provided. The analysed results are also examined by the Data Science Centre before exporting. Any results with fewer than 3 subjects are not allowed to be exported to prevent re-identification [22].

\section{CONCLUSION}

The NHIRD of Taiwan contains a large quantity of claims data and has the potential for multiple data linkages. Although more validation research is needed, and regulatory work to protect privacy is ongoing, this nationwide cohort is a valuable resource for medical research.

\section{ACKNOWLEDGEMENTS}

This work was financially supported by the Innovation and Policy Centre for Population Health and Sustainable Environment (Population Health Research Centre, PHRC), National Taiwan University College of Public Health from the Featured Areas
Research Centre Program within the framework of the Higher Education Sprout Project by the Ministry of Education (MOE) in Taiwan. We thank Professor Hsien-Ho Lin and Dr Yao-Hsu Yang for their valuable suggestions and information.

This study was supported by Pfizer Limited (TWPEHMed-2018-001), Ministry of Science and Technology (MOST-1073017-F-002-003), and the Featured Areas Research Center Program within the framework of the Higher Education Sprout Project by the MOE in Taiwan (grant no. NTU-107L9003).

\section{CONFLICT OF INTEREST}

The authors have no conflicts of interest to declare for this study.

\section{SUPPLEMENTARY MATERIALS}

Supplementary Material 1: Table S1 is available at http://www. e-epih.org/.

Supplementary Material 2: Table S2 is available at http://www. e-epih.org/.

\section{ORCID}

Liang-Yu Lin: http://orcid.org/0000-0003-4720-6738; Charlotte Warren-Gash: $h t t p: / / o r c i d . o r g / 0000-0003-4524-3180$; Liam Smeeth: http://orcid.org/0000-0002-9168-6022; Pau-Chung Chen: http:// orcid.org/0000-0002-6242-5974

\section{REFERENCES}

1. Hsing AW, Ioannidis JP. Nationwide population science: lessons from the Taiwan National Health Insurance Research Database. JAMA Intern Med 2015;175:1527-1529.

2. Gordis L. Epidemiology. 5th ed. Philadelphia: Elsevier; 2014,p. 163.

3. Eichler HG, Abadie E, Breckenridge A, Flamion B, Gustafsson LL, Leufkens $\mathrm{H}$, et al. Bridging the efficacy-effectiveness gap: a regulator's perspective on addressing variability of drug response. Nat Rev Drug Discov 2011;10:495-506.

4. Chen YC, Yeh HY, Wu JC, Haschler I, Chen TJ, Wetter T. Taiwan's National Health Insurance Research Database: administrative health care database as study object in bibliometrics. Scientometrics 2011;86:365-380.

5. Cheng TM. The Taiwanese health care system; 2017 [cited 2018 Nov 10]. Available from: https://international.commonwealthfund. org/countries/taiwan/.

6. National Health Insurance Administration. 2017-2018 National health insurance annual report. Taipei: National Health Insurance Administration, Ministry of Health and Welfare, Executive Yuan; 2017.

7. National Health Insurance Administration, Ministry of Health and Welfare, Taiwan. Universal health coverage in Taiwan; 2016 [cited 2018 Nov 10]. Available from: https://www.nhi.gov.tw/Resource/ 
webdata/21717_1_UnversalHealthCoverage-2.pdf.

8. National Health Insurance Administration Editorial Department. National health insurance holds your hands 12 years: growth and tranforamation. Taipei: National Health Insurance Administration; 2007, p. 30 (Chinese, author's translation).

9. National Health Insurance Research Database. Background; 2014 [cited 2018 Nov 10]. Available from: https://nhird.nhri.org.tw/en/ index.html.

10. Ministry of Health and Welfare Department of Statistics. Organization of Data Science Center 2018 [cited 2018 Nov 7]. Available from: https://dep.mohw.gov.tw/DOS/cp-2499-3563-113.html.

11. Health and Welfare Data Science Center. Data evaluation standard procedure. 2017 [cited 2018 Nov 15]. Available from: https:// dep.mohw.gov.tw/DOS/lp-2506-113.html (Chinese, author's translation).

12. Wu CY, Chen YJ, Ho HJ, Hsu YC, Kuo KN, Wu MS, et al. Association between nucleoside analogues and risk of hepatitis $\mathrm{B}$ virusrelated hepatocellular carcinoma recurrence following liver resection. JAMA 2012;308:1906-1914.

13. Kuo CF, Grainge MJ, Valdes AM, See LC, Luo SF, Yu KH, et al. Familial aggregation of systemic lupus erythematosus and coaggregation of autoimmune diseases in affected families JAMA Intern Med 2015;175:1518-1526.

14. Taiwan Biobank. Taiwan biobank annual issue. Taipei: Academia Sinica; 2017, p. 9 (Chinese, author's translation).

15. Ng AW, Poon SL, Huang MN, Lim JQ, Boot A, Yu W, et al. Aristolochic acids and their derivatives are widely implicated in liver cancers in Taiwan and throughout Asia. Sci Transl Med 2017;9: eaan6446.

16. Chen CJ, Yang YH, Lin MH, Lee CP, Tsan YT, Lai MN, et al. Herbal medicine containing aristolochic acid and the risk of hepatocellular carcinoma in patients with hepatitis B virus infection. Int J Cancer 2018. doi: https://doi.org/10.1002/ijc.31544.

17. National Taiwan University Health Data Research Center. Meeting minutes of NHIRD validation advisory board meeting; 2018 Aug 7; Taipei, Taiwan (Chinese, author's translation).

18. Supreme Administrative Court. Judgment-2017-No. 542017 [cited 2018 Nov 5]. Available from: http://jirs.judicial.gov.tw/FJUD/ index_1_S.aspx?p= Ig4rZO\%2BXdBODCjc\%2Bn1nm3YR5Dj5 RgDJ1wlyGJTejrL0\%3D (Chinese, author's translation).

19. Chen JH. Blockchain health is coming congressmen: medical records belongs patients; 2018 [cited 2018 Nov 15]. Available from: https://money.udn.com/money/story/5641/3218826 (Chinese, author's translation).

20. Health and Welfare Data Science Center. Health and welfare data science center data application; 2018 [cited 2018 Nov 5]. Available from: https://dep.mohw.gov.tw/DOS/lp-2499-113.html (Chinese, author's translation).

21. Ministry of Health and Welfare. 2017 Announcement for institutional review boards evaluation of qualifications; 2017 [cited 2018 Dec 20]. Available from: https://www.mohw.gov.tw/cp-18-389161.html (Chinese, author's translation).

22. Health and Welfare Data Science Center. Health and Welfare Ministry Data Science Center data exporting regulation; 2018 Oct 16 [cited 2018 Nov 5]. Available from: https://dep.mohw.gov. tw/DOS/cp-2498-44433-113.html (Chinese, author's translation). 